\title{
New Developments in Compositional Stage Mapping by EPMA and Micro-XRF
}

\author{
P. K. Carpenter ${ }^{1}$ and T.M. Hahn ${ }^{1}$ \\ ${ }^{1 .}$ Dept. of Earth and Planetary Sciences, Washington University in St. Louis, Campus Box 1169, Saint \\ Louis, MO, USA
}

Compositional mapping by wavelength-dispersive spectrometry (WDS) and electron-probe microanalysis (EPMA) can include sophisticated background subtraction and full matrix correction at each pixel to produce a concentration map. EPMA mapping coupled with backscattered-electron (BSE) imaging has superior spatial resolution and a mature correction algorithm, whereas micro-X-ray fluorescence ( $\mu \mathrm{XRF}$ ) mapping uses an efficient polycapillary optic with a larger fixed beam diameter and achieves a lower detection limits for selected elements. A fixed beam coupled with stage scanning to acquire a strip of X-ray intensity data is repeated over the map area. For both methods the per-pixel count time is 2-3 orders of magnitude shorter compared to spot analysis. High probe current is used for WDS EPMA mapping to compensate as current and time scale equally.

For this study, EPMA mapping runs were made on the WUSTL JEOL JXA-8200 with 5 wavelength-dispersive spectrometers (WDS) and a silicon-drift EDS, using JEOL and Probe Software applications. The X-ray intensity maps were acquired using Probe Image and Calcimage software, which performs a full $\Phi(\rho z)$ correction at each pixel in the map. The calibration uses a conventional quantitative analysis standardization, with WDS background subtraction made using the mean atomic number (MAN) background correction. The MAN background correction allows all counting time to be spent on the analytical peak, which significantly improves sensitivity during map runs. Two map passes were used to generate a 10 element WDS compositional map, with typical acquisition times of 12-24 hours, and 4 hours of computer time required for matrix correction of a 1024x1024 map.

The WUSTL EDAX Orbis $\mu$ XRF was used to compare with EPMA mapping. The $\mu$ XRF has a 30 micron spot size defined by a polycapillary optic, which improves low energy X-ray detection and reduces sampling of X-rays from deep within the sample. The Orbis has a silicon drift EDS detector and generates a spectrum image data cube which can be used for data mining. The map image resolution is defined using the 30 micron spot size, map size, and degree of sampling by the 30 micron beam. Typical compositional maps are acquired with dwell times of 50-500 msec, with map runs typically requiring 15-24 hours. The $\mu$ XRF can be used to map larger samples which benefit from being polished but do not need to be carbon coated.

A comb-layered xenolith is used to compare compositional mapping by EPMA and $\mu \mathrm{XRF}$. This sample is a $5 \times 6 \mathrm{~cm}$ rock slab containing multiple layers of mm-length dendritic plagioclase, sector-zoned Ca-pyroxene, euhedral to granular olivine, and quenched basaltic glass. It represents directional solidification during rapid cooling from a basaltic melt. Quantitative EPMA stage maps were acquired on a 1-inch round polished section using a $2.25 \mathrm{~mm}$ square map area at $800 x 800$ pixels, with analytical conditions of $15 \mathrm{kV}$ and $100-250 \mathrm{nA}$, a $3 \mu \mathrm{m}$ stage step size and beam diameter, and a dwell time of 150-500 msec per pixel. The WDS maps were processed as outlined above. The results are summarized in Fig. 1, which shows BSE and maps for major and trace elements. The Ca-pyroxene sector zoning is defined by small differences in chemistry, such as a Cr variation of $\sim 2000-5000 \mathrm{ppm}$. The analytical conditions of $37.5 \mathrm{nA}-\mathrm{sec}$ (i.e., $250 \mathrm{nA} * 0.15 \mathrm{sec}$ ) were necessary to measure $\mathrm{Cr}$ above the detection limit (DL); a conventional spot analysis at $50 \mathrm{nA}$ and $60 \mathrm{sec}$ count time is $300 \mathrm{nA}-\mathrm{sec}$ for comparison. The CalcImage DL map is useful for evaluating the compositional dependence of the DL due to different average $\mathrm{Z}$ of the phases. For the sector zoning there is a difference of 1220-1230 ppm DL for the two zones.

The $\mu \mathrm{XRF}$ was used to map a $21 \times 12 \mathrm{~mm}$ area on the comb-layer xenolith slab. This area is about an order of magnitude larger compared to the EPMA maps, and similar X-ray maps were produced. Because the $\mu$ XRF uses an EDS detector, one must inspect the EDS spectrum for pulse-pileup artifacts at higher count rates. Additionally, samples with large crystals can produce X-ray diffraction peaks which masquerade as characteristic peaks. The 
$\mu \mathrm{XRF}$ has very good sensitivity for higher energy X-rays such as $\mathrm{Sr} \mathrm{K} \alpha$ and is better for mapping of trace elements. Figure 2 illustrates the sum spectrum for a region at the top of the plagioclase layer that has elevated $\mathrm{Sr}$ content. This higher Sr content is evident in the $\mu \mathrm{XRF}$ map which uses the $\mathrm{Sr} \mathrm{K} \alpha$ line and samples a larger area, but was not observed by EPMA which necessarily uses the $\mathrm{Sr} L \alpha$ line. This underscores the need to evaluate samples on multiple spatial scales when considering the compositional variations.

In summary, both techniques have important advantages for compositional mapping and provide complementary data. We also present current efforts using Matlab, ENVI, and AXSIA to aid in analysis of mineral chemistry obtained by these compositional mapping methods.
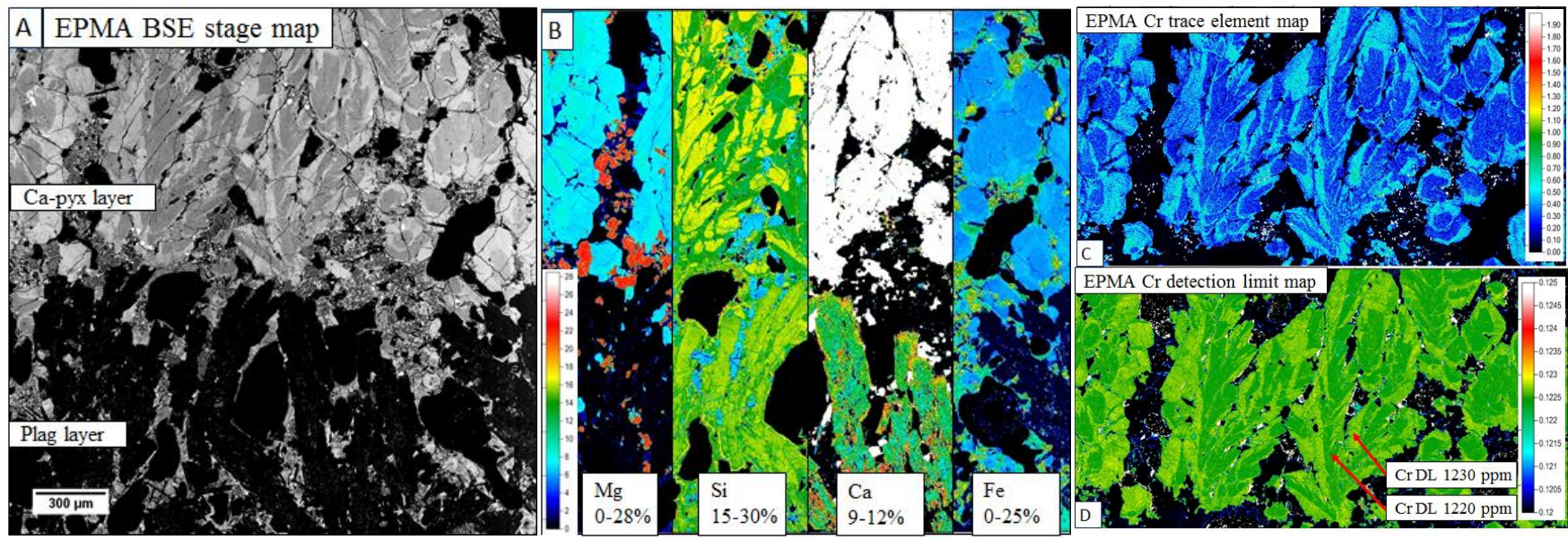

Figure 1. EPMA maps of comb-layer xenolith, all images from $2.25 \mathrm{~mm}$ square map area with 300 micron scale bar. (A) BSE stage map, sector-zoned Ca-pyroxene layer contains granular olivine, plagioclase layer has interstitial glass. (B) Quantitative concentration maps, color scale shown for $\mathrm{Mg}$ and range of element in wt\% shown on $\mathrm{Mg}, \mathrm{Si}, \mathrm{Ca}$, and $\mathrm{Fe}$ maps. Maps are in equal position to BSE map shown in (A). (C) Quantitative concentration map for $\mathrm{Cr}$ in Ca-pyroxene layer (upper half of BSE image (A)). Two sector compositions have 2000-5000 ppm concentration. Lower image shows $\mathrm{Cr}$ detection limit of $\sim 1220 \mathrm{ppm}$ at 3 -sigma with avg-Z dependence due to major-element sector chemistry.

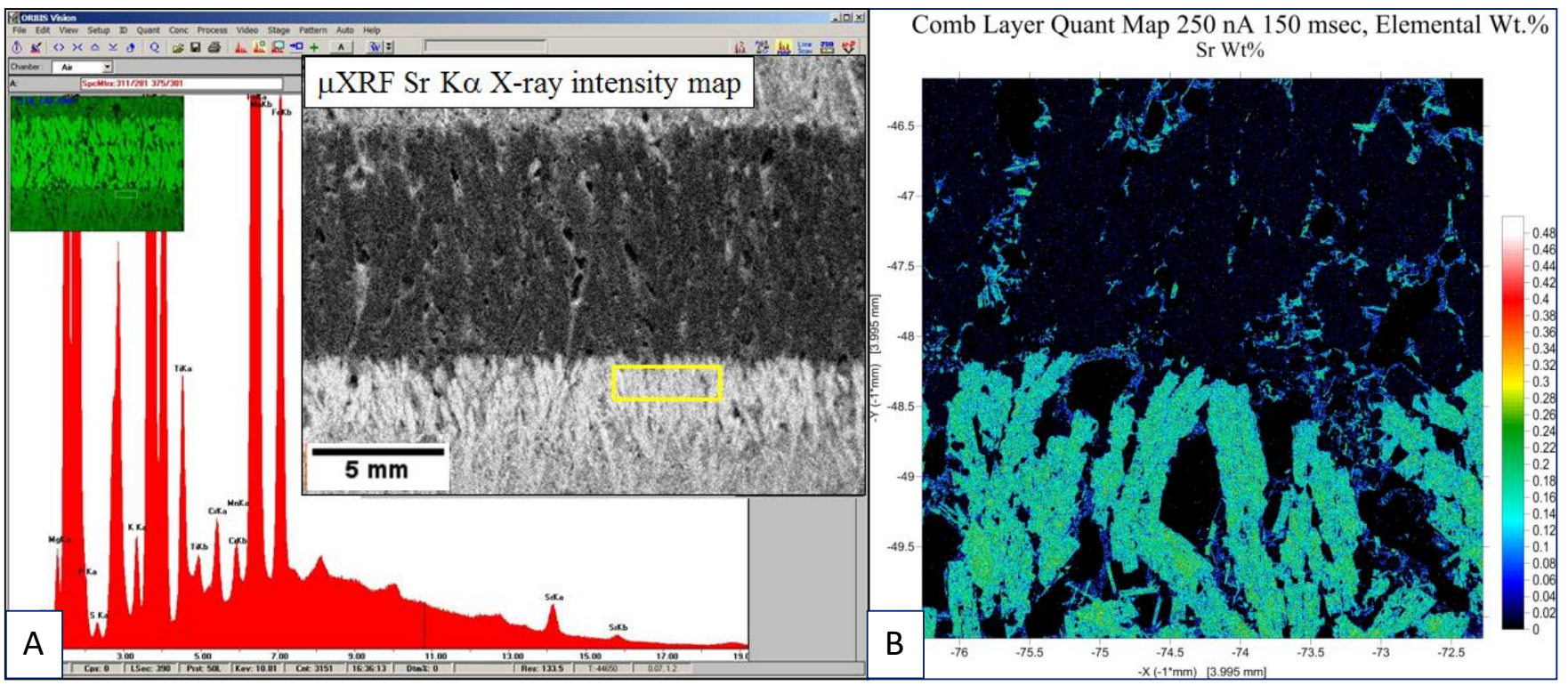

Figure 2. (A) Map from $21 \times 12 \mathrm{~mm}$ area of polished slab from same xenolith using $\mu$ XRF. EDS sum spectrum is derived from yellow region at top of plagioclase layer, and has peaks for both $\mathrm{Sr} K \alpha$ and $\mathrm{K} \beta$. The $\mu \mathrm{XRF}$ map was acquired at $512 \mathrm{x} 400$ pixels, $41 \mu \mathrm{m}$ step size with $500 \mathrm{msec}$ per pixel, and $600 \mu \mathrm{A}$ tube current at $30 \mathrm{kV}$. (B) Quantitative EPMA map for Sr which indicates the Sr level is approximately $2000 \mathrm{ppm}$ in the dendritic plagioclase. 